Case Report,

\title{
Limb Salvage Surgery of Osteosarcoma Usingliquid Nitrogen at Rsup Dr. Moh. Hoesin Palembang: A Case Series
}

\author{
Primadika Rubiansyah ${ }^{1}$, Afifurrahman ${ }^{2}$ \\ ${ }^{1,2}$ Departments of Sriwijaya University Indonesia \\ Email Address: afifurrahman_rizal@yahoo.com
}

\begin{abstract}
:
The five-year survival rate of osteosarcoma has gradually increased to $60 \%$ from $20 \%$ with Limb Salvage Surgery (LSS) combined with chemotherapy. Limb salvage surgery involves two main steps, wide resection of the bone tumor, followed by reconstruction of the defect. Reconstruction can be achieved with Cryosurgery.

In this study, we presented a serial case of four patient at RSUP Dr. Moh. Hoesin Palembang with ages between 13-19 years old, 2 male and 2 females and diagnosed with Osteosarcoma Enneking stage IIB at the Lower Extremity Region that treated with Cryosurgery from October 2020- February 2021.

The cryosurgery procedure on RSUP dr. Moh Hoesin Palembang, started with the bone soaked into liquid Nitrogen for 20 minutes. The bone than thawed at room temperature for 15 minutes and then soaked with normal saline in room temperature for another 15 minutes. The used of Cryosurgery for limb salvage surgery shown a good result for inducing osteosarcoma necrosis with all Histopathology results shown 5090\% to $91-99 \%$ post cryosurgery (Huvos grade II-III).

The followed up however shown mixed results, with the patients have mixed knee flex ranged from 40o to 1100, but The LLD could still be preserved at 1-2 cm. During Followed up at 1-2 month postoperative, we found complication such as failure of fixation and skin necrotic. The followed-up research also still needed for long termed results, complications and for assessing the recurrency rated of the Osteosarcoma for these cases.
\end{abstract}

Keywords: Osteosarcoma, Limb Salvage Surgery, Cryosurgery, Liquid Nitrogen.

\section{Interoduction:}

Osteosarcoma is the most common primary malignant bone tumor of the long bones, with children and adolescents at particular risk. Osteosarcoma is the most common primary bone sarcoma but less than $1 \%$ of all cancer cases ${ }^{1,2}$,for bone and soft tissue malignancies involving the extremities until the1970s. Prior to 1970, the treatment of Osteosarcoma depended primarily on surgical resection, resulting in 5-year survival rates below $20 \%$. The advances in radiological imaging and the increased effective use of adjuvant treatment, both chemotherapy and irradiation, share the credit for increasing successful limb salvage surgery. The five-year survival rate of osteosarcoma has gradually increased to $60 \%$ from $20 \%$, and the limb salvage rate has significantly increased $^{1,3}$
Limb salvage surgery (LSS) techniques aim to widely excise of the tumor at the margins of healthy tissue. If this cannot be achieved, amputation is indicated. The type of surgery is determined based on tumor location and size, extramedullary extension, presence of metastatic disease, initial tumor necrosis, age and skeletal development. LSS combined with chemotherapy is the preferable choice of osteosarcoma's treatment by the majority of surgeons. ${ }^{4,5}$

Limb salvage surgery involves two main steps, wide resection of the bone tumor, followed by reconstruction of the defect. Reconstruction can be achieved with the use of non-biological endoprosthesis or biological methods such as allograft, vascularized fibula, recycled autograft or by making use of a combination of allograft/autograft and endoprosthesis. Excellent 
survival rates and long-term good results have been reported for this method of reconstruction. However, there are concerns regarding long-term durability of endoprosthesis on account of complications like loosening, fracture, failure of locking mechanism and infection which often requires revision $3,6,7$

Tsuchiya et al describes a reconstruction method using tumour-bearing autograft treated by liquid nitrogen. While in the surgery, the excised bone was frozen in liquid nitrogen for 20 minutes, thawed at room temperature for 15 minutes, further thawed in distilled water for ten minutes and finally re-implanted with instrumentation or composite use of a prosthetic replacement. As this has been mainly developed in Asia countries, there are still not many reports published about this procedure. $^{8,9}$ In this study, we presented a serial case of four patient in RSUP Dr. Moh. Hoesin Palembang with diagnosed Osteosarcoma of Lower Extremity Region that treated with Cryosurgery from October 2020- February 2021.

\section{Case Presentation:}

There are 4 cases of patient with diagnoses of Osteosarcoma of the lower limb from October 2020 - February 2021 that was treated with Cryosurgery with ages between 13-19 years old, 2 male and 2 females. All patient came with the same symptoms with lumps on the lower limbs that gotten bigger, with pain and difficult to move their lower limbs. The Laboratories findings of the patients also shown increase of Alkaline Phosphatase Level. The Histopathology results of all the patients was Osteosarcoma. All the patient has been treated with Neoadjuvant chemotherapy and shown shrinkage of the tumours.

The First patient, SC/M/19yo came with the lumps around his left knee (Figure 1) with difficulty to extend his knee. There was no venectation and distal edema from this patient that suggest there was no infiltration to his vascularization. The results of the Xray, and MRI (figure 2 and 3) finding supported the diagnoses findings of Osteosarcoma that extend to the knee joint (Enneking Stage IIB). The result of biopsy was Osteosarcoma at the distal femur.

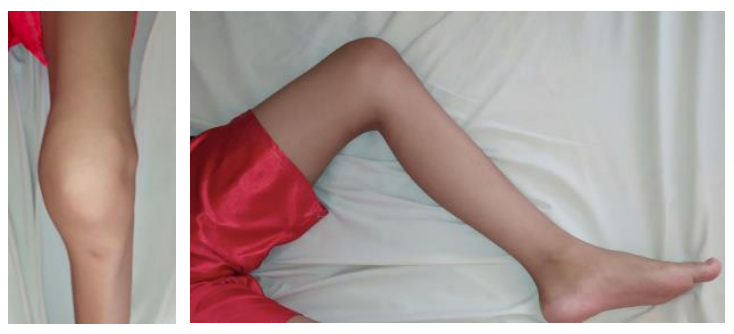

Figure1 . SC/M/19yo with lump on his left knee

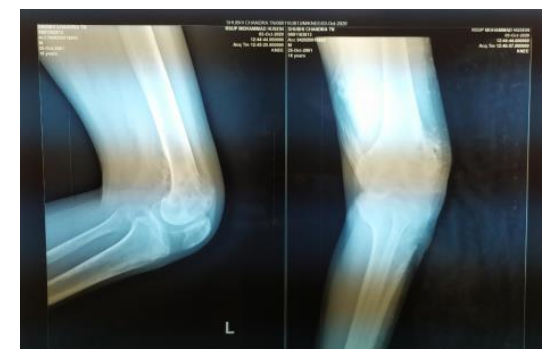

Figure2. Xray knee joint AP/Lat
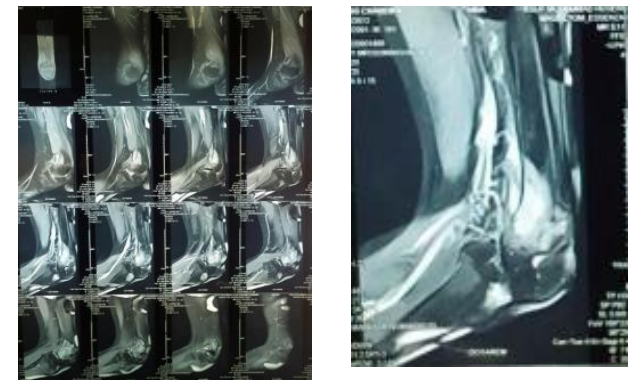

Figure3. MRI of the knee joint

At $22^{\text {nd }}$ October 2020, the patient than treated with Limb Salvage Surgery with cryosurgery techniques. We performed Wide Excision of the tumours mass, then the tumour mass was excised from the bone part of distal femur (figure 4). The bone than soaked into liquid Nitrogen for 20 minutes. The bone than thawed at room temperature for 15 minutes then soaked with normal saline in room temperature for another 15 minutes. Part of the bone before and after soaked to the liquid nitrogen than sent to the Pathological anatomy department for examination.

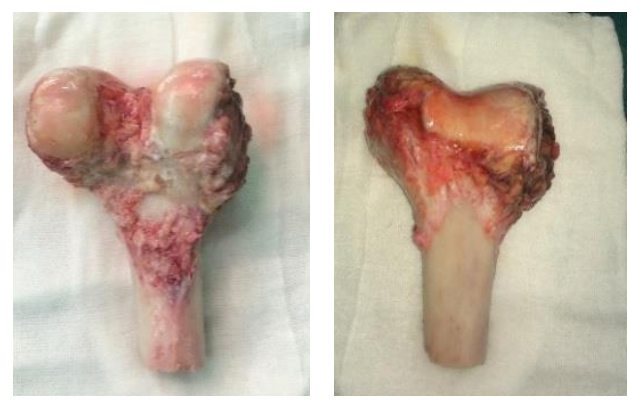

Figure4. Tumor Distal femur 
After the Cryosurgery, the bone than fixed with internal fixation with plate and screws, and the knee joint underwent Total Knee Replacement. (Figure 5 and 6).

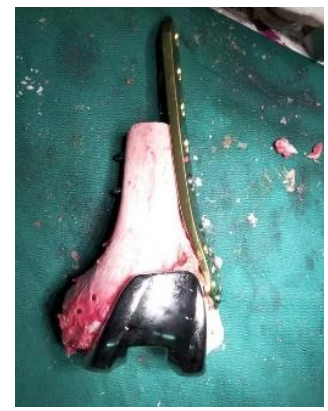

Figure5. Post Cryosurgery and reconstruction
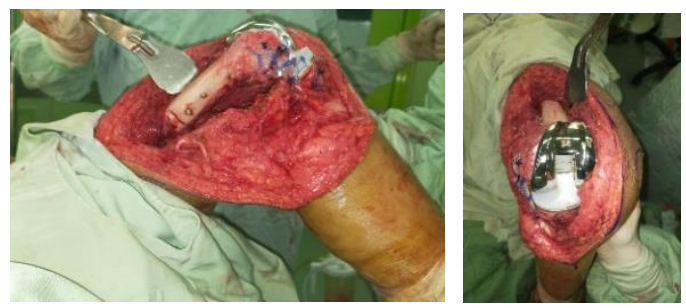

Figure6. Distal femur and knee joint reconstruction, with plate and screw and total knee replacement

The Xray postoperative result shown a good alignment. (Figure 7A) during follow up 2-month post-operative the Xray still shown good alignment (Figure 7B). The result of the Histopathology examination of the patients shown area of necrosis $0-50 \%$ pre cryosurgery (Huvos grade I) and 5090\% post cryosurgery (Huvos grade II) (Figure 7C and 7D). Followed up for this patient 3-month post operation shown a good result with the patient able to walk with crutches and flexed his knee for $90^{\circ}$ with LLD $1 \mathrm{~cm}$ (Figure 7E)
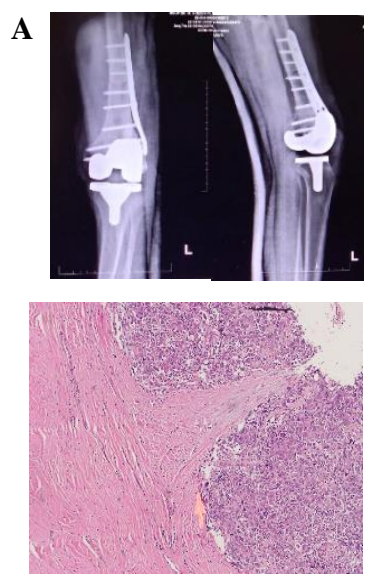

C
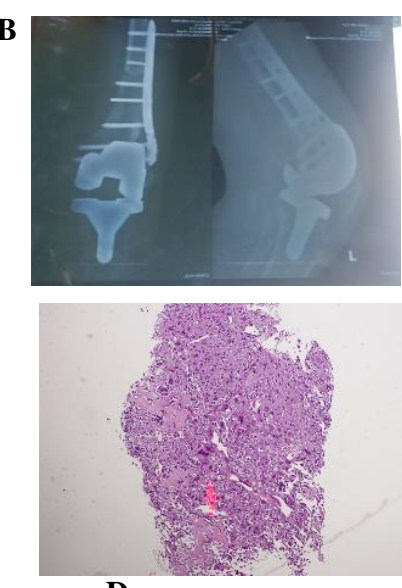

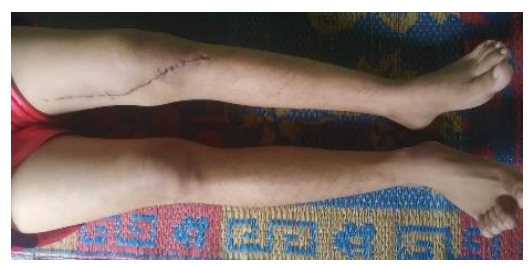

Figure7. XRay Genu Kiri AP/Lat (A) post Operation (B) 2 Month post Operation (C) Histopathology pre cryosurgery (D) post cryosurgery (E) Patient follow up 3month post operation

The second patient RK/M/13yo came with lump from his right thigh that gotten bigger in the last 6 months before admitted to the hospital there was also history of fall on his right thigh. From examination there was pain and difficulty to bend the knee due to the lump. There was no venectation and distal oedema from his right limb that suggest there was no infiltration to his vascularization. The results of the Xray, and MRI (figure 9 and 10) finding supported the diagnoses findings of Osteosarcoma that extend to extra compartment soft tissue without involvement of epiphyseal plate (Enneking Stage IIB). The core biopsy has been done with resultant of Osteosarcoma at the distal femur.

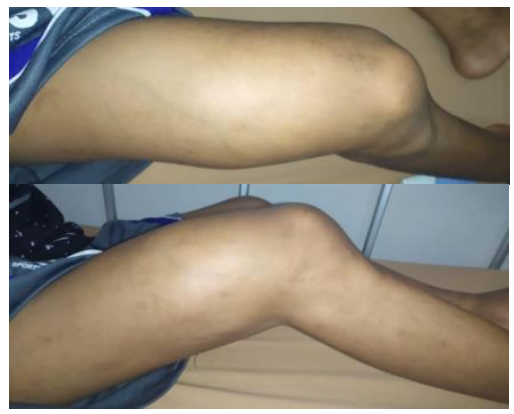

Figure8. RK/M/13yo lump on his right thigh

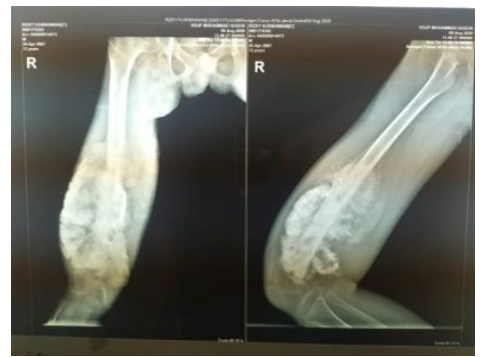

Figure9. Xray of right femur AP/Lat
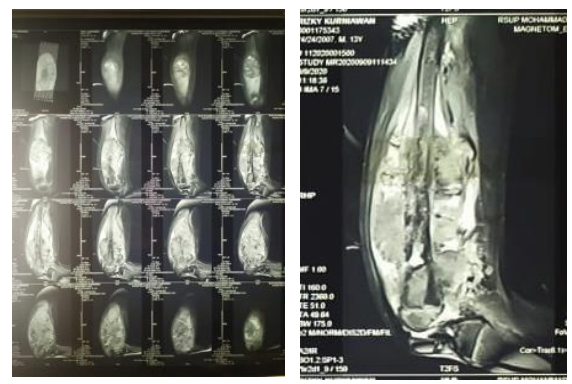

Figure10. MRI of right femur 
At $12^{\text {th }}$ November 2020 , the patient than treated with Limb Salvage Surgery with cryosurgery techniques. We performed Wide Excision of the tumours mass, then the tumour mass was excised from the bone part of distal femur (figure 11A). The bone than soaked into liquid Nitrogen for 20 minutes (Figure 11B). The bone than thawed at room temperature for 15 minutes then soaked with normal saline in room temperature for another 15 minutes. Part of the bone before and after soaked to the liquid nitrogen than sent to the Pathological anatomy department for examination. The distal part of the femur bone fixed to the proximal part with plate and screw than fixed to the condylar part of the femur with tension band wire (Figure $12 \mathrm{~A}$ and $\mathrm{B})$.

A
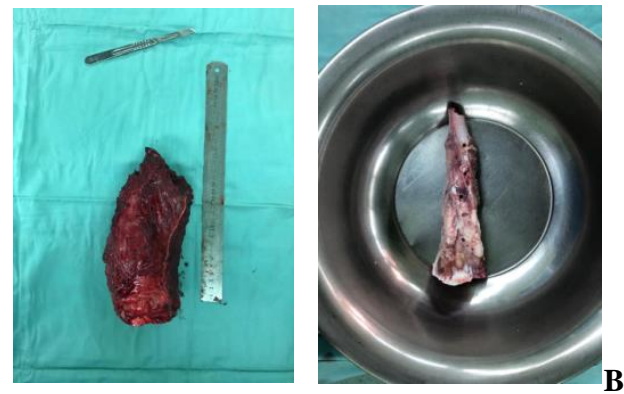

Figure11. (A) Tumor specimen (B) Tumor after cryosurgery

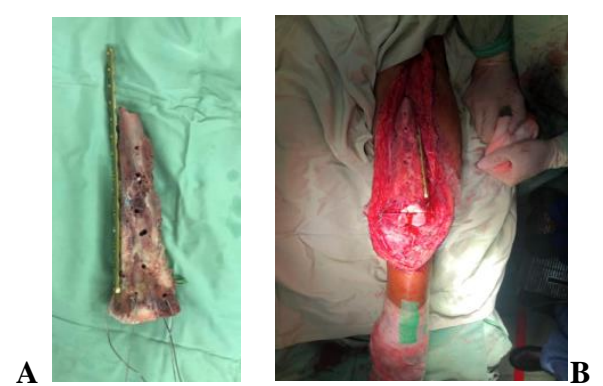

Figure 12 (A) Fixation with plate and screw

(B) Fixation with Tension Band Wiring

The Xray postoperative result shown a good alignment. (Figure 13A), but 2-month postoperative shown an implant failure (Figure 13B). The result of the Histopathology examination of the patients shown area of Osteosarcoma necrosis around $0-50 \%$ pre cryosurgery (Huvos grade 1) and $50-91 \%$ post cryosurgery (Huvos grade II) (Figure 13C and 13D). Unfortunately, during Followed up 2-month postoperative the patient was fall down and got failure of fixation in distal part, the patient able to flexed his knee for $40^{\circ}$ with LLD: $2 \mathrm{~cm}$.
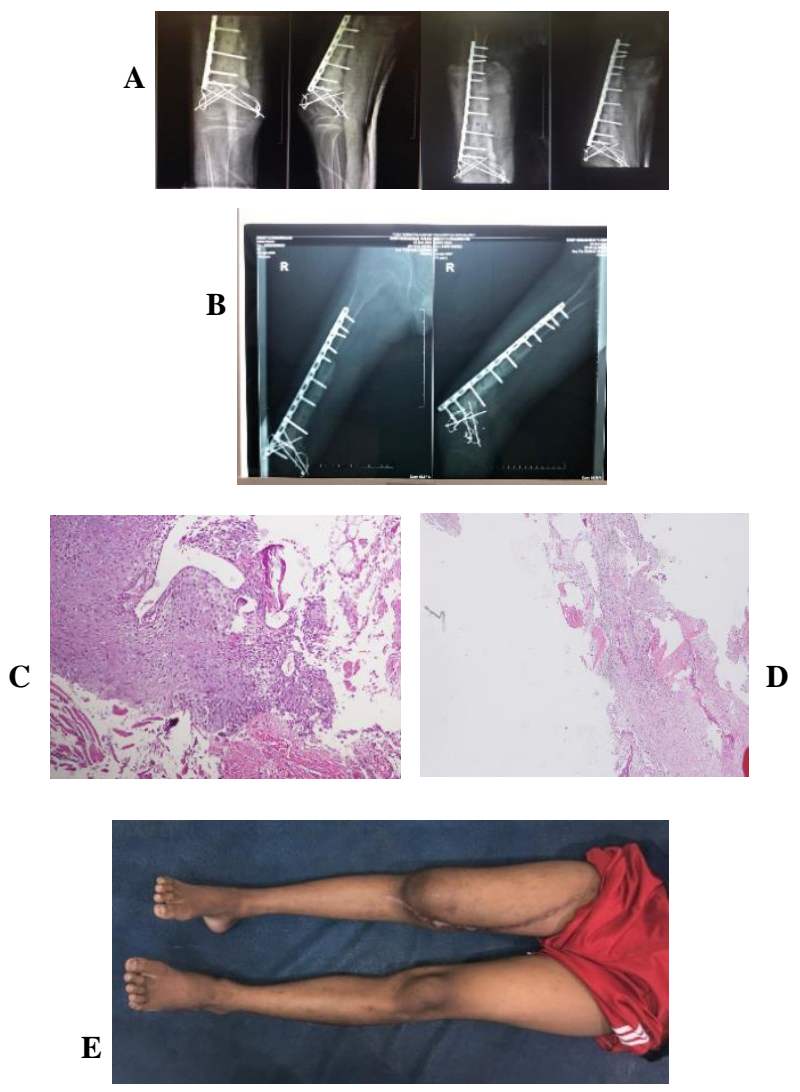

Figure13. Xray Right Femur (A) Post Cryosurgery (B) Follow up Xray 2 Month Post Cryosurgery (C)

Histopathology pre cryosurgery

(D) Post cryosurgery (E) Patient follow up 2-month post operation

The third cases, CA/F/15yo with lump from his right Leg that gotten bigger in the last 6 months. From examination there was pain and difficulty to bend the knee due to the lump. There was no venectation and distal oedema from her right limb that suggest there was no infiltration to her vascularization. (Figure 14) The results of the Xray, and MRI (figure 15 and 16) finding supported the diagnoses findings of Osteosarcoma of proximal right tibia that extend to extra compartment soft tissue and extend to the knee joint (Enneking Stage IIB). The core biopsy has been done with resultant of Chondroblastic Osteosarcoma.

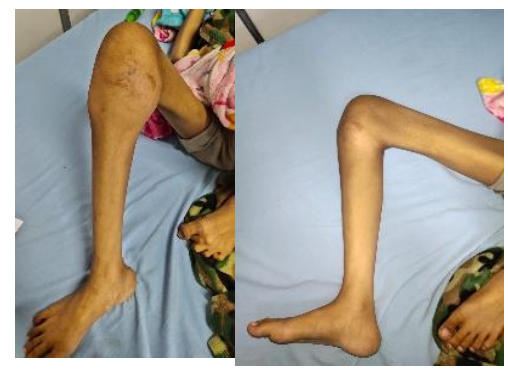

Figure14. CA/F/15yo lump on his right Le 


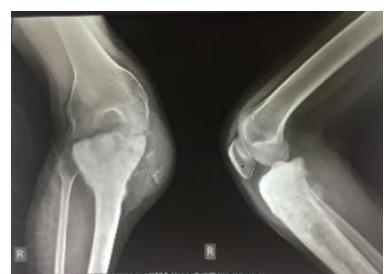

Figure15. Xray of right knee AP/Lat
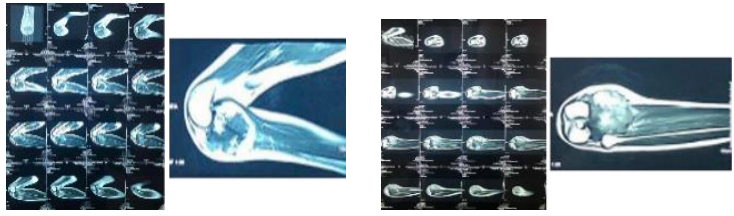

Figure16. MRI of right tibia

At $28^{\text {th }}$ January 2021, the patient than treated with Limb Salvage Surgery with cryosurgery techniques. We performed Wide Excision of the tumours mass, during this process there were some Rupture of the peroneal artery, we performed repair artery with end to end sutured. Then the tumour mass was excised from the bone part of Proximal (figure 17A). The bone than soaked into liquid Nitrogen for 20 minutes (Figure 17B). The bone than thawed at room temperature for 15 minutes then soaked with normal saline in room temperature for another 15 minutes. Part of the bone before and after soaked to the liquid nitrogen than sent to the Pathological anatomy department for examination. The distal part of the tibia bone than fixed to the distal part with plate and screw and Total Knee Replacement (Figure 18 A). The anterior part of tibia than covered with Medial gastrocnemius flap to close the wound (Figure 18B)

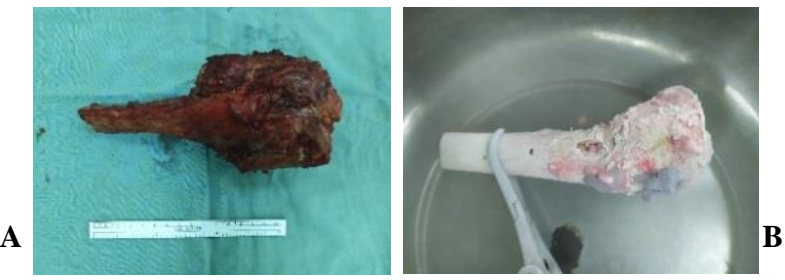

Figure17. (A) Tumor specimen (B) Tumor after cryosurgery

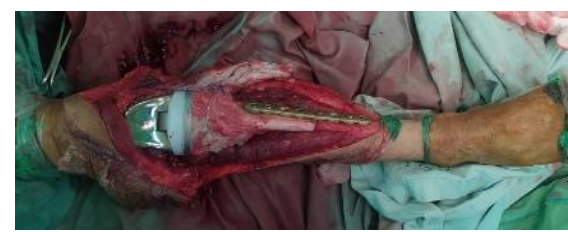

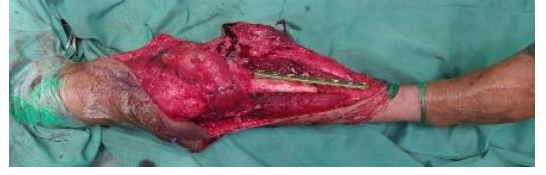

Figure18 (A) Fixation with plate and screw and TKR (B) Medial Gastrocnemius Flap

The Xray postoperative result shown a good alignment. (Figure19 A). The result of the Histopathology examination of the patients shown area of necrosis for Osteosarcoma pre cryosurgery $0-50 \%$ (Huvos grade 1) and post cryosurgery 5090\% (Huvos grade II) (Figure $19 \mathrm{~B}$ and C). Unfortunately, during Followed up for this patient 1-month postoperative result shown some skin necrotic above the medial gastrocnemius. During follow up the patient can flex the knee until $40^{\circ}$ with LLD: 1cm (Figure 19 D)
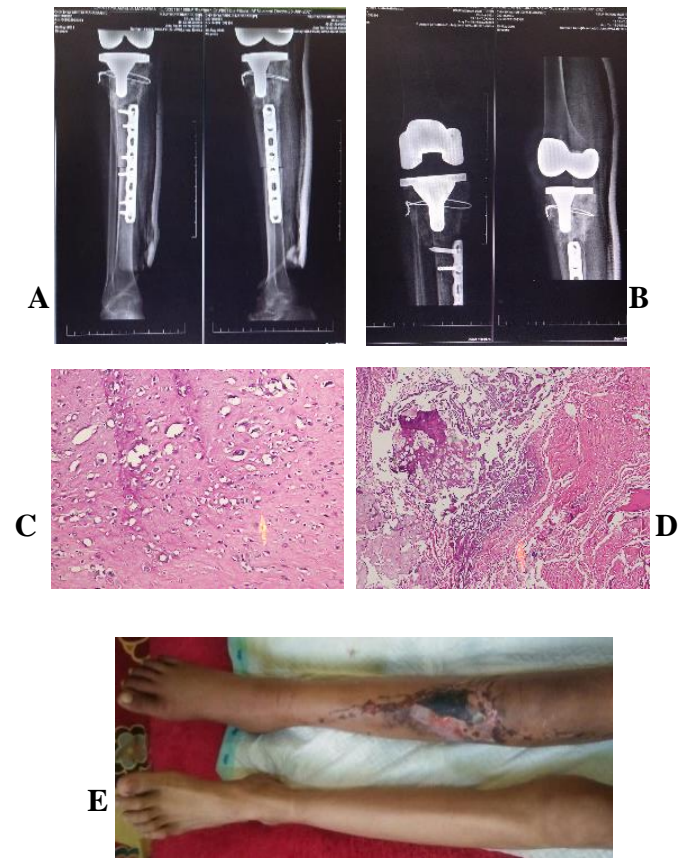

Figure19 (A) Xray Right Femur Post Cryosurgery (B) Histopathology pre cryosurgery (C) post cryosurgery (D) Follow up 1 month post Cryosurgery

The last cases, $\mathrm{A} / 13^{\text {th }} / \mathrm{F}$ Came with lump on her right thigh in the past 6 months before admitted to the hospital. From examination there was pain and difficulty to bend the knee due to the lump. There was also scar from biopsy from previous hospital. There was no venectation and distal oedema from her right limb that suggest there was no infiltration to her vascularization. (Figure 20) The results of the Xray, and MRI (figure 21 and 22) finding supported the diagnoses findings of Osteosarcoma of midshaft femur that extend to extra compartment soft tissue (Enneking Stage IIB). The 
core biopsy has been done with resultant of Chondroblastic Osteosarcoma.
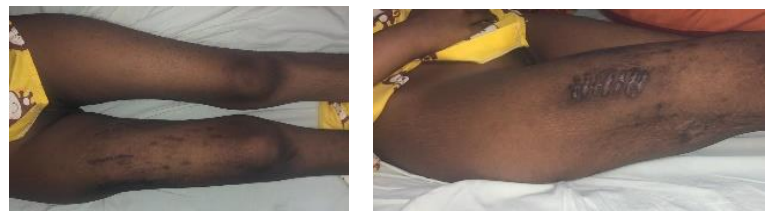

Figure20. $\mathrm{A} / 13^{\text {th }} / \mathrm{F}$ lump on his right thigh

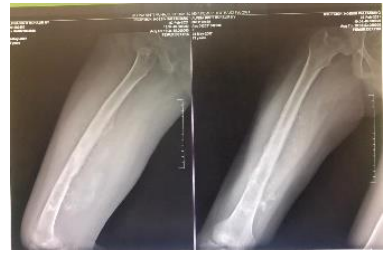

Figure21. XRay Pre Op (3-2-2021)

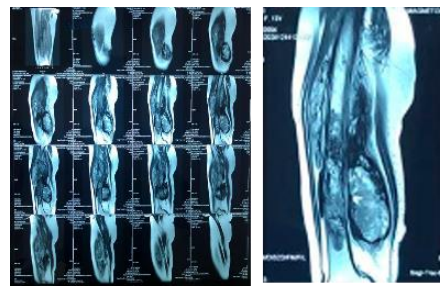

Figure22. MRI Pre Op (14-12-2020)

At $4^{\text {th }}$ February 2021, the patient than treated with Limb Salvage Surgery with cryosurgery techniques. We performed Intercalary resection of the tumours mass. Then the tumour mass was excised from the bone (figure 23). The bone than soaked into liquid Nitrogen for 20 minutes. The bone than thawed at room temperature for 15 minutes and then soaked with normal saline in room temperature for another 15 minutes. Part of the bone before and after soaked to the liquid nitrogen than sent to the Pathological anatomy department for examination. The bone than fixed with distal femur LCP (16 holes) and screw (Figure 23).

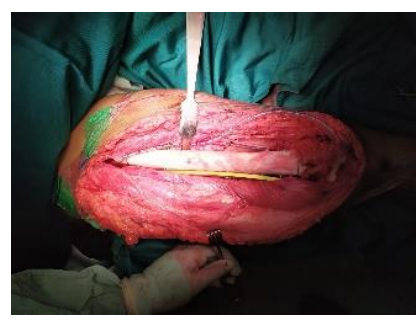

Figure24. Post Cryosurgery and Fixation with plate and screw

The Xray postoperative result shown a good alignment. (Figure $25 \mathrm{~A}$ ). The result of the Histopathology examination of the patients shown area of osteosarcoma necrosis around $0-50 \%$ pre cryosurgery (Huvos grade 1) (Figure $25 \mathrm{~B}$ ) and 9199\% post cryosurgery (Huvos grade III) (Figure 25 C). During followed up 1-month post-surgery this patient shown good result with good alignment of the bone, and the patient able to flexed her knee for $110^{\circ}$ with LLD: $1 \mathrm{~cm}$ (Figure $25 \mathrm{D}$ and $\mathrm{E}$ ).

A
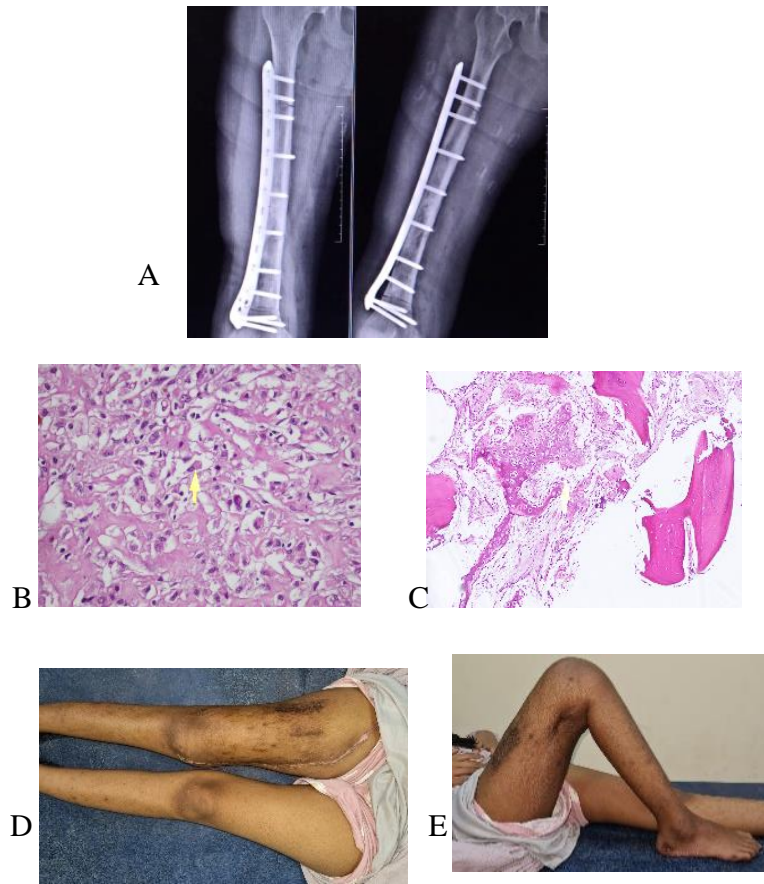

Figure25. XRay Right Femur (A) Post Cryosurgery (B) Histopathology pre cryosurgery (C) post cryosurgery (D) Follow up 1 month post Cryosurgery (E) The patient able to bent her knee for $110^{\circ}$

\section{Discussion:}

The end results from the limb salvage from the first to fourth patient shown a mixed result, with some shown satisfactory results, with others shown some complication during followed up. All Xray postoperative result shown a good alignment and the result of the Histopathology examination of the All the patients post cryosurgery shown area of 
osteosarcoma necrosis around 50-90\% and 91-99\% (Huvos grade II-III).

The followed up however shown mixed results, with the first patient shown a good result with the patient able to walk with crutches and flex his knee for $90^{\circ}$ with LLD: $1 \mathrm{~cm}$. The second patient unfortunately, during Followed up 2-month postoperative, patient was fall down and got failure of fixation on distal part. The patient able to flex his knee for $40^{\circ}$ with LLD: $2 \mathrm{~cm}$. With fracture as one of the most common complication of the cryosurgery, this result was still expected. ${ }^{10}$

The Third patient during 1-month postoperative followed up shown skin necrotic above the medial gastrocnemius flap. The patients felt pain on her anterior leg, and unwilling to flexed the knee. During passive flex of the knee, the end result was $40^{\circ}$ with LLD: $1 \mathrm{~cm}$. For the fourth patient, during followed up 1-month post-surgery the patient able to willingly flex her knee for $110^{\circ}$ with LLD: $2 \mathrm{~cm}$. The cryosurgery procedure on RSUP dr. Moh Hoesin Palembang, started with the bone tumour soaked into liquid Nitrogen for 20 minutes. The bone than thawed at room temperature for 15 minutes and then soaked with normal saline in room temperature for another 15 minutes. This procedure is the same with research by Tsuchiya, et al 2005. ${ }^{9}$ Another procedure by Chen $\mathrm{C}$, et al 2017, states that Cryosurgery may be as effective as wide resection for therapeutic treatment of benign-aggressive, low grade, and malignant bone tumors with a slow freeze and quick thaw can preserve cells, but a quick freeze and slow thaw is repeated to induce cell death. Exposing the curettage area to a quick freeze below $-20{ }^{\circ} \mathrm{C}$ creates ice crystals that disrupt cell osmolality which leads to apoptosis. A minimum of 2 freeze thaw cycles is needed to achieve a level of necrosis comparable to other adjuvant therapies, with 3 cycles having no significant difference in apoptosis. However, choosing to use excessive amounts of freeze thaw cycles has been associated with higher fracture rates and non-unions. Therefore, two freeze cycles appear to balance necrosis while limiting complications. ${ }^{10}$ this procedure slightly difference with the procedure at RSUP dr. Moh Hoesin Palembang where we use only one freeze cycles.

\section{Summary:}

In summary the used of Cryosurgery for limb salvage surgery shown a good result for inducing osteosarcoma necrosis with Histopathology results shown $50-90 \%$ and $91-99 \%$ (Huvos grade II-III) post cryosurgery. There are mixed results shown at the followed up, with complication liked failure of fixation and skin necrosis. Suggesting further research. The followed-up research also still needed for long termed results, complications and for assessing the recurrency rated of the Osteosarcoma for these cases.

\section{Reference:}

[1] Yang Y, Han L, He Z, Li X, Yang S, Yang J, Zhang Y, Li D, Yang Z. Advances in limb salvage treatment of osteosarcoma. 2018. Journal of Bone Oncology. 10: 36-40.

[2] Liu C, Shu C. Liquid nitrogen for cryotherapy treatment for osteosarcoma of the middle femur: A case report. Journal of Clinical Laboratory Analysis. 2021: 1-5.

[3] Rahman MA, Bassiony A, Shalaby H. Reimplantation of the resected tumourbearing segment after recycling using liquid nitrogen for osteosarcoma. 2009. International Orthopaedics, 33; 1365-1370.

[4] Papakonstantinou E, Stamatopoulos A, Athanasiadis DI, Kenanidis E, Potoupnis M, Haidich AB, Tsiridis E. Limb-salvage surgery offers better five-year survival rate than amputation in patients with limb osteosarcoma treated with neoadjuvant chemotherapy. A systematic review and meta-analysis. Journal of Bone. 2020; 25.

[5] Zhang SP, Li X, Li H, Sun XH, Yan XF. Significance of Neoadjuvant Chemotherapy (NACT) in Limb Salvage Treatment of Osteosarcoma. European Review for Medical and Pharmacological Sciences. 2018; 22: 6538-6544.

[6] Garg SK, Aggarwal P, Virk J, Punia RPS, Dimri K, Jindal R. Limb Salvage Using Liquid Nitrogen-Treated Tumour-Bearing Autograft: A Single Institutional Experience of 10 Patients. Indian Journal of Orthopaedics. 2020; 54:200-207.

[7] Kinkel S, Lehner B, Kleinhans JA, Jakubowitz E, Ewerbeck V, Heisel C. Medium to long-term results after reconstruction of bone defects at the knee with tumour endoprosthesis. Journal of Surgical Oncology. 2010;101: 166-169

[8] Yazawa Y, Imanishi J, Torigoe T. Limb Salvage Using Liquid Nitrogen Treated Autologous Bone Graft in Sarcoma Surgery. 
Japan Journal of Medicine. 2018; 1(5): 239242.

[9] Tsuchiya H, Wan SL, Sakayama K, Yamamoto N, Nishida $\mathrm{H}$, Tomita $\mathrm{K}$. Reconstruction using an autograft containing tumour treated by liquid nitrogen. The
Journal of Bone and Joint Surgery. 2005; 87: 218-225

[10] Chen C, Garlich J, Vincent K, Brien E. Postoperative complications with cryotherapy in bone tumors. Journal of Bone Oncology. 2017; 7: 13-17. 